Pensamiento Crítico N. ${ }^{\circ}$ 14, pp. 119-135

\title{
El Mercado Integrado Latino Americano (MILA). Contexto, Hipótesis y Reflexiones
}

Carlos Palomino Selem

\section{RESUMEN}

La integración y fusión de bolsas tiene como propósito aumentar la participación de estas dentro del mercado internacional de inversionistas, hoy bastante competitivo. Pero la efectividad que tenga estas integraciones dependerá de que los costos de transacción resulten competitivos; el proceso de integración no será de inmediato, los inversionistas se toman un tiempo en movilizar capitales de una bolsa a otra, se trata de un problema de información y familiaridad de los activos de las otras bolsas. La ventaja del MILA es que los países que la componen tienen un perfil similar.

Palabras clave: Fusión, mercado al mostrador, comisión de bolsa y títulos, mercado de negociación electrónica, sistema de red electrónica de negociación, broker que negocia en mercado electrónico, flujo de capitales por liquidez.

\begin{abstract}
The integration and merger of the stock exchanges have the propose to increase their participation in the international or regional capital markets; but this will be possible if the stock exchange's transaction costs are competitive respect to others markets. The MILA is an interesting experience because the integration imply three
\end{abstract}




\section{Carlos Palomino Selem}

countries that have similar economic performance, but it will be necessary that the economic model continue without modification in the member countries.

Keywords: Merger, over the counter, security and exchange commission, electronic trading market, electronic communication network system, institutional broker, flight to liquidity.

\section{INTRODUCCIÓN}

El entorno de los negocios bursátiles ha cambiado sustantivamente en la primera década del presente siglo. Este se ha caracterizado por la creciente internacionalización de las bolsas, principalmente, de aquellas ubicadas en los centros financieros internacionales de Estados Unidos de América y Europa.

En este contexto, en América Latina se está constituyendo una primera experiencia de internacionalización, en la cual se encuentra involucrada la Bolsa de Valores de Lima (BVL). En efecto, el carácter de la mencionada internacionalización, corresponde a la integración de tres bolsas localizadas en América del Sur, a la cual se denominada como Mercado Integrado Latinoamericano (MILA). Las bolsas en mención son las siguientes: la Bolsa de Colombia, la Bolsa de Santiago de Chile y la Bolsa de Valores de Lima (BVL).

En el presente artículo, primero se presentaran los principales esquemas de internacionalización que involucran experiencias de bolsas ubicadas en centros financieros, específicamente las de Nueva York: La Bolsa de Valores de Nueva York (New York Stock Exchange: NYSE) y el Nasdaq (National Association of Securities Dealers Automated Quotation). En segundo término, explicar de qué trata el MILA. En tercer lugar, nos remitiremos al contexto macroeconómico en que inicialmente se da el surgimiento del MILA. En el cuarto punto, se expondrá un estado del conocimiento respecto a las motivaciones y los resultados producto de las fusiones bursátiles de los 2000. Por último, propondremos un conjunto de hipótesis respecto a los resultados que traería del MILA para la BVL. 


\section{ESQUEMA DE LA INTERNACIONALIZACIÓN DE LOS MERCADOS BUR- SÁTILES}

El medio ambiente de los negocios bursátiles ha experimentado cambios sustanciales en la última década. La tendencia en esta ha sido llevar a las bolsas de forma creciente hacia mayores niveles de internacionalización.

Entendemos por internacionalización al hecho de que una bolsa se fusione (merger) con una o más bolsas de otros países, regiones o continentes, con el propósito de una mayor participación en los mercados bursátiles del exterior, con la finalidad de minimizar su exposición a la competencia.

En este sentido, dos importantes experiencias resultan interesantes esquematizar. La primera, proviene del hoy Nasdaq-OMX.; la segunda, tiene su origen en la adquisición de Archipiélago por parte del NYSE.

A continuación pasaremos a presentar el esquema de las mencionadas fusiones, lo que debe quedar en claro es que no pretendemos hacer una historia de ellas.

\subsection{El Nasdaq- OMX}

El Nasdaq-OMX es la fusión de dos grupos; por una parte, del Nasdaq Group; por otra parte, del OMX.

\subsubsection{La formación de Nasdaq}

El Nasdaq (NASDAQ:NDAQ), fundada por la National Association of Securities Dealers (NASD) ${ }^{1}$, fue inicialmente un mercado de tipo over the counter ${ }^{2}$, un tercer mercado; es decir, un mercado no organizado ${ }^{3}$, pero que la Securities and Exchange Commission (SEC) autorizó su funcionamiento como un mecanismo de negociación electrónica (Electronic Trading), con el propósito de contribuir a la transparencia en las operaciones de mercado, así, el Nasdaq empezó a operar en febrero de $1971^{4}$.

1 http://es.wikipedia.org/wiki/Nasdaq.

2 CFA Institute; Textos de Enseñanzas para Preparación al Examen CFA- Nivel 1.

3 Se trata de un mecanismo de negociación no centralizada, poco autorregulado, pero sí supervisado por la autoridad correspondiente del mercado.

4 http://es.wikipedia.org/wiki/Nasdaq. 


\section{Carlos Palomino Selem}

La importancia de Nasdaq radicó en que sentó la base para el posterior desarrollo del concepto de la negociación electrónica financiera de tipo bursátil que posteriormente se denominó Electronic Communication Network (ECN).

En efecto, el aporte de mayor trascendencia que trajo consigo la ECN a la esfera bursátil, y que hasta hoy perdura, se puede observar en múltiples aspectos que fueron los siguientes: eficiencia para operar de forma profunda (compras y ventas a distintos precios en cada instante) títulos, alta exactitud y transparencia en la administración de los precios y sus órdenes, bajo riesgo operativo, gran alcance geográfico, y una significativa reducción en los costos de transacción.

El año 2000, luego de experimentar una profunda reestructuración, Nasdaq se convirtió en una compañía con fines de lucro propiedad de Nasdaq Stock Market.

\subsubsection{El Nasdaq Group: la primera gran fusión}

En su objetivo de obtener mayor participación en el mercado accionario de los Estados Unidos y abarcar a un nuevo tipo de inversionista, en el año 2005 Nasdaq Stock Market compró a su competidor más importante, Instinet Group Inc., un Broker Institucional ${ }^{5}$, cuyo dominio representaba más de 40 mercados globales y 1500 clientes institucionales ${ }^{6}$. Con esta adquisición, Nasdaq superó al NYSE en número de acciones listadas.

La razón de ser de la compra radicaba en la adquisición del mecanismo electrónico de negociación (una Electronic Trading Network: ECN) considerada la más potente de aquel entonces, lo cual hacía de Nasdaq el mercado más líquido en relación al resto de las bolsas americanas, incluyendo al NYSE.

\subsubsection{El OMX y la fusión con Nasdaq Stock Market}

Por su parte, el OMX se conformó el año 2003 por la fusión de seis (6) bolsas de origen sueco-finesas:

5 Broker Institucional se refiere a un bróker que principalmente organiza un mercado en torno a fondos de inversión. Ver: http://www.investopedia.com/terms/i/instinet.asp

6 http:// www.nasdaq.com/newsroom/stats/performance 
La Bolsa de Copenhague

La Bolsa de Estocolmo

La Bolsa de Helsinki

La Bolsa de Tallinn (ex URSS)

La Bolsa de Riga (ex URSS)

La Bolsa de Vilna (ex URSS)

Finalmente, en el año 2001 se consolidó la fusión del Nasdaq Stock Market y el OMX, constituyéndose una importante red de tipo electrónica mundial que extendía su alcance hacia varias bolsas del norte de Europa, al otro lado del Atlántico; organizándose en tres tipos de mercados que clasificaron a su interior con los nombres siguientes: el Global Select Market, el Global Market y el Capital Market. Estos mercados listan más de 3900 compañías y 3073 emisores.

\subsection{La Bolsa de Nueva York: El NYSE Group}

La Bolsa de Nueva York (NYSE:NYA) es considerada la bolsa de valores más grande del mundo, su capitalización bursátil (market capitalization) fue de USD 13.39 trillones ${ }^{7}$ a diciembre del 2010.

Anticipándose al Nasdaq Stock Market, el NYSE, inició el proceso de adquisición de un ECN denominado Archipiélago Holfings (ArcaEx año 2001), la misma que se consolidó en diciembre del 2005. En marzo del 2006, ya con el ArcaEx, el NYSE fortaleció su sistema de negociación híbrido, que combina la negociación en piso (floor) con el mecanismo de negociación electrónica.

En abril del 2007, el NYSE dio otro paso en su proceso de expansión, fusionándose con Euronext, un mercado integrado europeo. Así, al igual que Nasdaq-OMX, el NYSE cruzó el Atlántico.

Euronext fue el primer mercado integrado europeo, se constituyó en septiembre del 2000 con las bolsas de París, Amsterdam, Bruselas, Lisboa y Oporto, ingresando a esta unión la inglesa LIFFE, una importante bolsa especializada en derivativos de futuros y opciones.

7 http://www.world-exchanges.org/statistics/time-series/market-capitalization 


\section{Carlos Palomino Selem}

\section{EL MERCADO INTEGRADO LATINO AMERICANO (MILA)}

El MILA es el primer mercado bursátil integrado, no una fusión, de tres bolsas de América Latina, que comprende los mercados organizados de Perú, Colombia y Chile; uno de sus objetivos consiste en el fortalecimiento institucional del mercado bursátil de cada país miembro. Al igual que las mencionadas fusiones del NYSE-Euronext y el Nasdaq-OMX busca ganar una mayor participación en los mercados bursátiles; lo cual implica objetivos de liquidez de mercado, profundidad y reducción de costos de transacción con transparencia y calidad.

El perfil de indicadores bursátiles del MILA al inicio del proceso, en términos de capitalización bursátil, número total de operaciones (variable de profundidad) y monto negociado, resultan ser menores en comparación a las importantes bolsas latinoamericanas como la de Brasil y México (ver Gráfico 1, 2 y 3).

\section{FUNDAMENTOS MACROECONÓMICOS PARA LA INTEGRACIÓN DEL MILA}

\subsection{El modelo económico seguido}

La integración requiere de un marco microeconómico y macroeconómico que corresponda a un modelo conceptual y de gestión compartido. El desempeño económico de los países miembros ha resultado muy similar en los últimos años, generando una coyuntura propicia para concretar la integración; esperando que los cambios de gobiernos, que sustentan a las democracias, mantenga la continuidad del modelo económico que han venido sosteniendo los países miembros.

En efecto, en esta dirección, el modelo económico seguido por los países miembros es el de economía de mercado, las elecciones en Colombia no ha variado en este sentido la orientación, y se espera que después de las elecciones de abril del 2011 en Perú sigan el mismo camino; es decir, microeconómicamente considerar el mercado y el sistema de precios que se deriva de este como el principal mecanismo de asignación de recursos económicos, sin la intervención empresarial del Estado y la implementación de políticas populistas; asignando al Estado un rol de regulador prudente. 


\section{el Mercado Integrado Latino Americano (MILA). Contexto, Hipótesis y Reflexiones}

\subsection{El perfil macroeconómico}

Macroeconómicamente, los tres países han sostenido un crecimiento económico por encima del promedio de la región, reduciendo la volatilidad ${ }^{8}$ de este (ver Gráfico 4), manteniendo el balance en sus cuentas fiscales (ver Gráfico 5) y la estabilidad monetaria expresada en un comportamiento del nivel de precios aceptable para el mercado.

En términos de inflación, los tres países trabajan en base al esquema conocido de Metas Explícitas de Inflación, que asumieron de manera independiente cada uno por su parte a inicios década de los 2000. Este esquema adopta las bandas sobre las cuales pueden fluctuar los precios. Similar esquema emplea, también, El Sistema de la Reserva Federal de Estados Unidos de América (su banco central: el Fed).

La importancia de aplicar este esquema consiste en que permite a la autoridad monetaria coordinar explícita o implícitamente la política de tasas de interés de referencia entre bancos centrales; lo cual es la forma moderna de regular el nivel de actividad económica y la rentabilidad de los activos monetarios y financieros entre países, una manera de prevenir movimientos desequilibradores por parte de los flujos de capitales de corto plazo entre estos.

En el Cuadro 1 se muestran las metas explícitas de inflación establecidas por los países miembros del MILA; observamos que estas son iguales entre Chile y Colombia, y comportan un nivel semejante al de Perú, aunque algo por encima de este. El Gráfico 6 presenta la evolución de la inflación, que en los años recientes se ha venido estabilizando.

Respecto al riesgo país, lo cual tiene un efecto directo sobre el costo de las emisiones, tanto soberanas como las privadas pertenecientes a compañías localizadas en la plaza de cada uno de los países miembros, esta ha experimentado una significativa reducción (ver Gráfico 7 y Cuadro 2), donde muy posiblemente el año 2011 Colombia alcance grado de inversión igualando a Perú y Chile en este aspecto.

Otro factor de riesgo, el cual ha traído problemas en algunos países de la Eurozona, ha sido el nivel de endeudamiento del país y la evolución de la deuda soberana; en este sentido, el grupo de países del MILA expresan un desempeño algo variado; por

8 A excepción del año 2009, por efecto de la crisis financiera mundial del 2008. 


\section{Carlos Palomino Selem}

ejemplo, el perfil de la deuda bruta total/\%PBI en Perú ha ido mejorando en los últimos años, aunque se encuentra a distancia de Chile; pero, en el caso de Colombia, requerirá mayor trabajo (ver Gráfico 8), aunque podemos juzgar, en términos generales, que la evolución en este indicador ha seguido una tendencia vista como positiva.

En cuanto a la relación deuda de largo plazo/\%Reservas Internacionales, importante indicador de riesgo de mercado, este ha mostrado una evolución positiva en todos los miembros del MILA (ver Gráfico 9); el mencionado indicador, se interpreta como el porcentaje que representa la ejecución de la carga de largo plazo, si se pagara todo hoy, respecto a la caja disponible del país en divisa en el momento actual.

\section{MARCO CONCEPTUAL SOBRE LAS FUSIONES Y/O ADQUISICIONES BURSÁTILES}

Las investigaciones llevadas a cabo respecto a las fusiones bursátiles surgieron por la preocupación que causaba los costos de capital de las emisiones realizadas en los mercados primarios, la cual, se vería afectada por los problemas de liquidez en los mercados bursátiles. Estudios de Amihud \& Mendelson (1986) que estiman la relación entre la liquidez y el valor de las acciones, así como los de Brennan \& Subrahmanyam (1996) y Datar, Naik y Radcliffe (1998) que relacionaron los retornos de las acciones con diversos indicadores de liquidez, tienen como principal preocupación el tema de la liquidez en los mercados secundarios.

Entre los trabajos más recientes y relevantes sobre los resultados de las fusiones se encuentra la investigación de Nielsson $(2009)^{9}$; Nielsson, examinó los resultados obtenidos en la importante fusión entre el Grupo NYSE y Euronext, que comentamos en el segundo punto del presente trabajo.

Nielsson concluye, que en la fusión del NYSE con Euronext (NYSE-Euronext), se destaca la presencia de una asimetría en términos de liquidez en beneficio de las acciones de las grandes firmas corporativas reconocidas a nivel mundial, respecto a las medianas y pequeñas firmas que operan para el mercado doméstico y no son de predominante perfil exportador. Según Nielsson, incluso aún se puede generar lo que se

9 Nielsson, desarrolló su tesis doctoral de la cual se publicó una parte en el Journal of Financial Markets. 


\section{EI Mercado Integrado Latino Americano (MILA). Contexto, Hipótesis y Reflexiones}

denomina como un flight to liquity; esto significa, que la liquidez migra hacia los mercados y las acciones, que antes de la fusión, eran las más líquidas y conocidas. El tiempo de aprendizaje que toma para que los inversionistas se informen sobre las compañías menos conocidas en los mercados menos líquidos, determinará el tiempo que tomará el retorno de los capitales hacia los mercados menos líquidos y la mejora del valor de los títulos de las firmas medianas y pequeñas.

El efecto flight to liquidity llevó a realizar estudios como los de Pagano (1989) y Choudhry \& Nanda (1991) respecto a los costos de transacción y el rol que desempeñan en el direccionamiento de los flujos de capitales hacia las bolsas. Una conclusión importante se encuentra en la determinación de la relación directa que existe entre bolsas o mecanismos de negociación más líquidos y la eficiencia en términos del desarrollo de los sistemas operativos, por un lado; y por otro, los menores costos de transacción en dichos mercados.

Por tanto, se puede concluir, que dos factores afectarían la liquidez de los mercados en una fusión: 1) la rigidez que exista en las preferencias de los inversionistas por reasignar sus portafolios hacia los mercados foráneos, de los cuales se tiene poca información; 2) los menores costos de transacción que incentivan la concurrencia de inversionistas hacia mecanismos más eficientes.

\section{HIPÓTESIS SOBRE POSIBLES RESULTADOS DEL MILA}

De acuerdo a lo expuesto anteriormente, podemos intentar algunos posibles resultados que podrían alcanzarse con el MILA.

\section{Hipótesis $\mathrm{N} .^{\circ}$ 1: El MILA absorberá capitales financieros provenientes de las AFP del Perú hacia los otros países miembros.}

Esta afirmación es falsa, porque los flujos financieros de largo plazo se movilizan primero, hacia países que presentan estabilidad política y un modelo de crecimiento económico de carácter neoliberal en su esencia; en segundo término, interesa las empresas y proyectos que presentan un perfil de rentabilidad requerido por los inversionistas. En este sentido, el MILA podría generar un flujo de capitales hacia Perú. 


\section{Carlos Palomino Selem}

\section{Hipótesis $\mathrm{N} .^{\circ}$ 2: El MILA traería mayor liquidez hacia la Bolsa de Valores de Lima (BVL).}

Esta afirmación dependerá del plazo que se tome y los costos de transacción. En el corto plazo, el flujo puede ser menor que en el largo plazo. En el corto plazo, la rigidez por parte de los inversionistas por comprar empresas registradas en la BVL, pero cuyos nombres no son reconocidos internacionalmente, retardaría un mayor flujo de recursos líquidos; y por tanto, la liquidez experimentaría un crecimiento menor al que se podría esperar.

En el largo plazo, una mayor información sobre performance y oportunidad de inversión en las empresas registradas en la BVL incrementaría la liquidez en la bolsa local respecto al corto plazo.

\section{Hipótesis $\mathbf{N} .^{\circ}$ 3: El MILA dará mayor liquidez a las acciones menos líquidas de la BVL.}

Esta hipótesis se deriva de la anterior (N. $\left.{ }^{\circ} 2\right)$. Esto no es necesariamente verdad. La evidencia empírica en otras bolsas demuestra que las empresas menos beneficiadas por la liquidez son aquellas que no desarrollan mercados en el exterior y se convierten en reconocidas mundialmente.

\section{Hipótesis $\mathrm{N}^{\circ}$ 4: El MILA permitirá alinear los precios de los títulos que se negocian en las bolsas miembros.}

En esta parte, el tema resulta más complejo; por un lado, se encuentra lo relacionado a los productos similares; por otro lado, el relativo a los costos de transacción.

En lo relacionado a los productos similares, las bolsas miembros se caracterizan por constituirse en mercados donde predomina en el índice una estructura sectorial diferente.

En este caso, la alineación de precios se debe fundamentar en los múltiplos, particularmente en el ratio Precio / Utilidad por Acción (P/E); el mismo que puede interpretarse como el precio que paga un inversionista por la utilidad por acción. En esta 
dirección, los $\mathrm{P} / \mathrm{E}$ de los índices representativos del mercado y los sectoriales debieran tender hacia una convergencia.

Respecto a los costos de transacción, es necesario que estos se igualen, lo cual implica un trabajo de tipo institucional, entre los costos de operar una u otra bolsa; y el otro, el tratamiento del impuesto al valor agregado (IVA) o como le denominamos en Perú, el impuesto general a las ventas.

\section{Hipótesis $\mathrm{N} .^{\circ}$ 5: El MILA requiere de políticas monetarias y fiscales coordi- nadas para evitar una desventaja entre mercados bursátiles.}

El MILA requiere que las políticas monetarias y fiscales sean coordinadas. En efecto, este es un aspecto que poco se ha discutido. El tratamiento monetario y fiscal requiere coordinación con dos propósitos: por un lado, para evitar distorsiones en las rentabilidades de empresas, ganancias de capital y dividendos; otro lado, para ajustar un comportamiento cíclico lo más uniforme posible, de manera que las políticas trabajen en desventaja de una bolsa con respecto a otra.

\section{BIBLIOGRAFÍA}

Amihud, Y. and L.H. Mendelson (1986). "Asset pricing and the bid-ask spread", Journal of Financial Economics, 17, 223-249.

Brennan, M.J. and A. Subrahmanyam. (1996). "Market microstructure and asset pricing: On the compensation for illiquidity in stock returns". Journal of Financial Economics, 41, 441-64.

Chowdhry, B. and V. Nanda (1991). "Multimarket trading and market liquidity", The Review of Financial Studies, 4, 3.

CFA Institute; Textos de Enseñanzas para Preparación al Examen CFA- Nivel 1.

Datar, V., N. Naik and R. Radcliffe (1998). "Liquidity and stock returns: An alternative test", Journal of Financial Markets, 203-19.

http://es.wikipedia.org/wiki/Nasdaq; mayo 2011. 


\section{Carlos Palomino Selem}

http://www.investopedia.com/terms/i/instinet.asp; mayo 2011.

http:// www.nasdaq.com/newsroom/stats/performance; mayo 2011

http://www.world-exchanges.org/statistics/time-series/market-capitalization; mayo 2011.

Inteligo SAB (2011). "Entorno Macroeconómico MILA"; document de trabajo.

www.bloomberg.com; mayo 2011.

Ulf Nielsson (2009). "Stock exchange merger and liquidity: The case of Euronext"

Journal of Financial Markets, Volume 12, 229-267. 


\section{ANEXOS}

Capitalización Bursátil al 31 dic. 2010 1,545,566 (Millones de US\$)

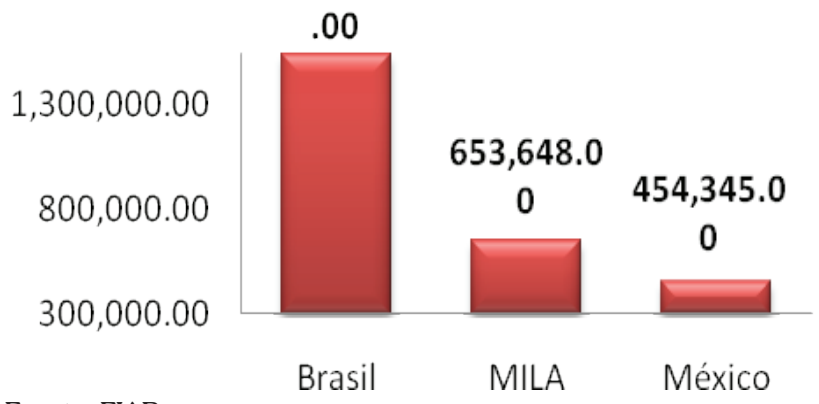

Fuente: FIAB

Gráfico 1

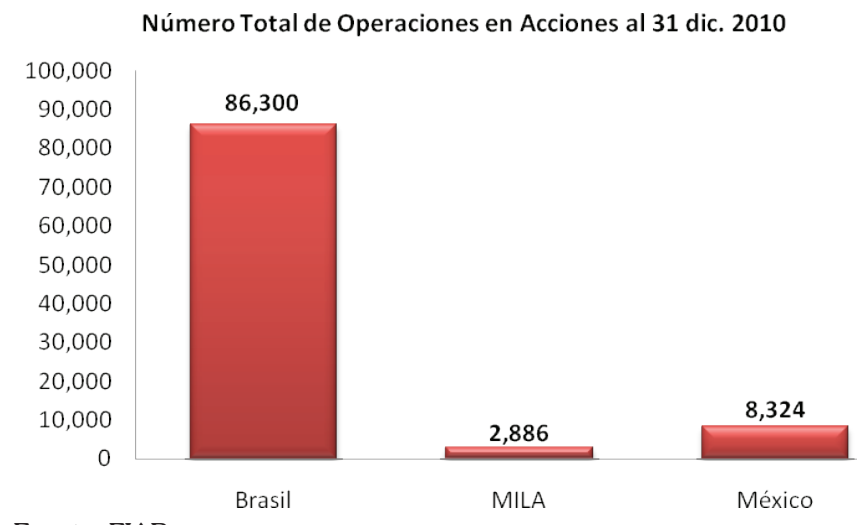

Gráfico 2 


\section{Carlos Palomino Selem}

Monto Negociado en Acciones al 31 dic. 2010

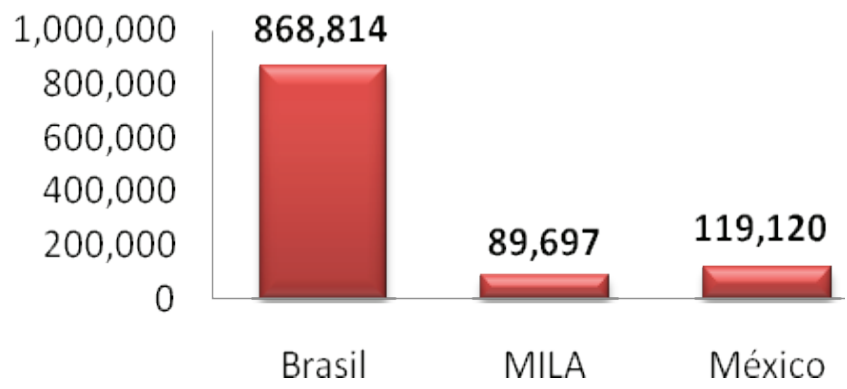

Fuente: FIAB

Gráfico 3

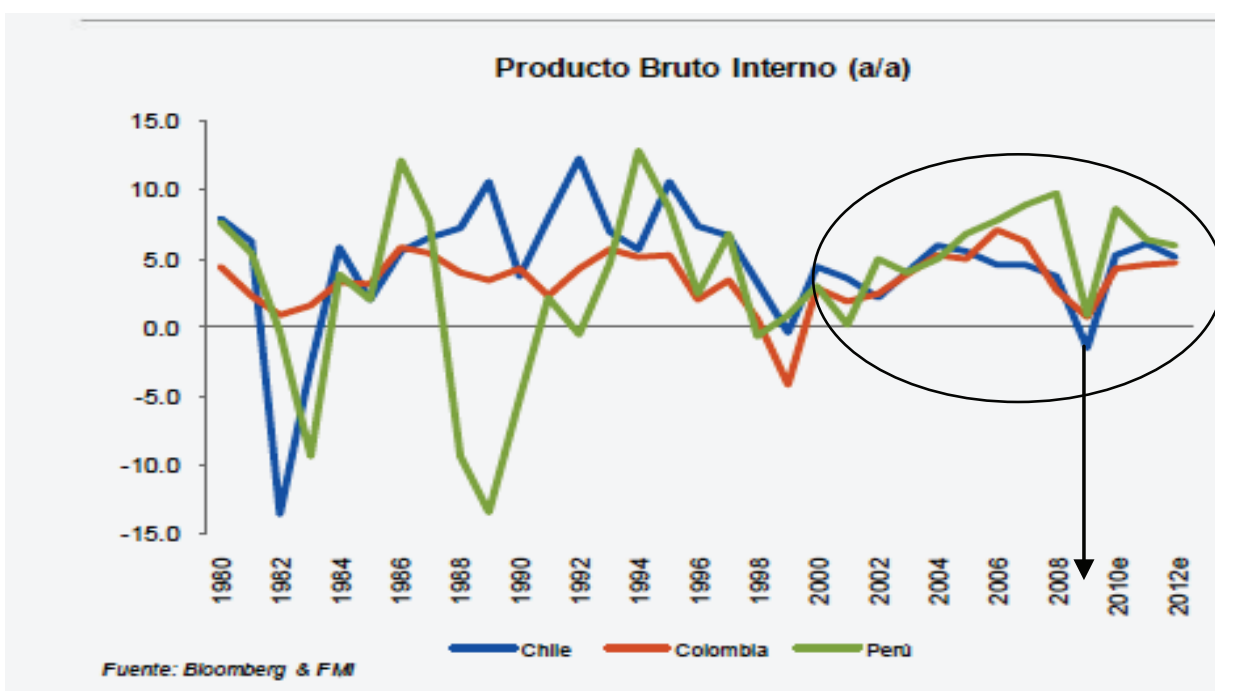

Elaboración: Propia.

Gráfico 4 


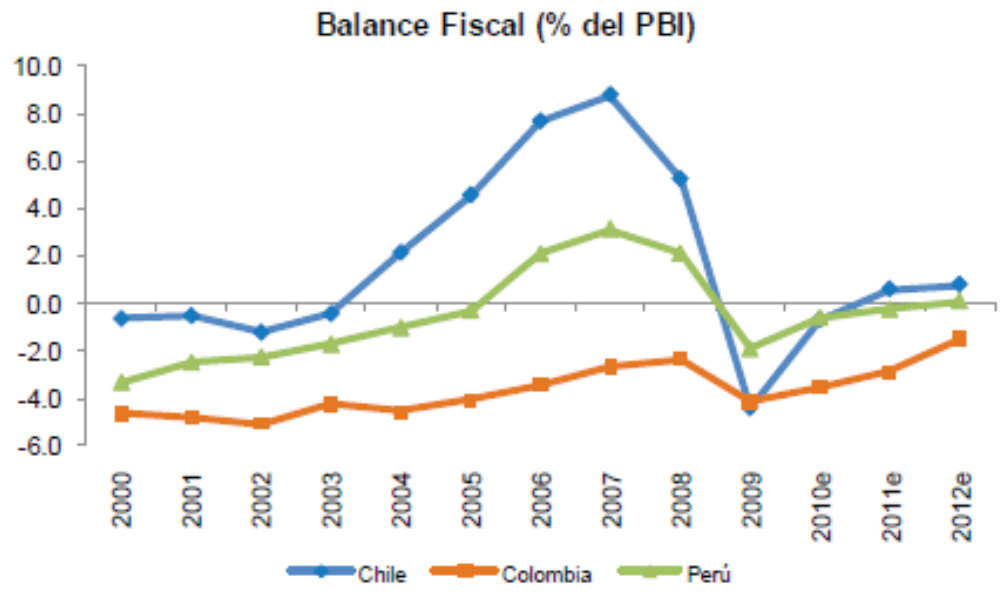

Elaboración: Propia.

Gráfico 5

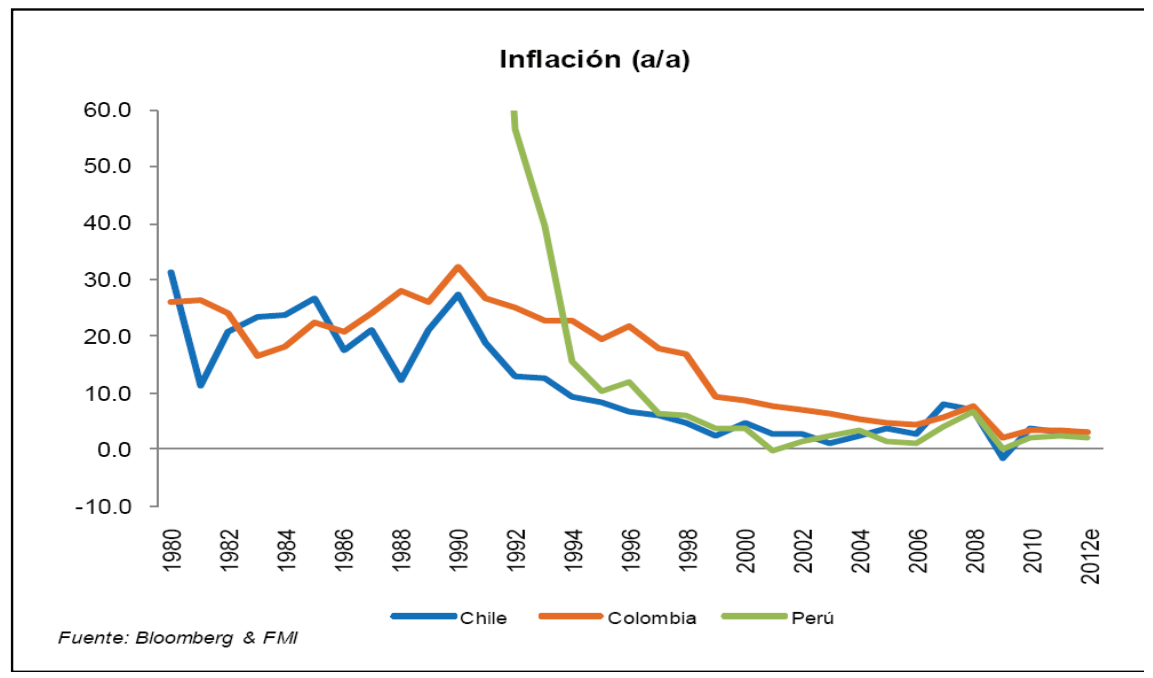

Gráfico 6 


\section{Carlos Palomino Selem}

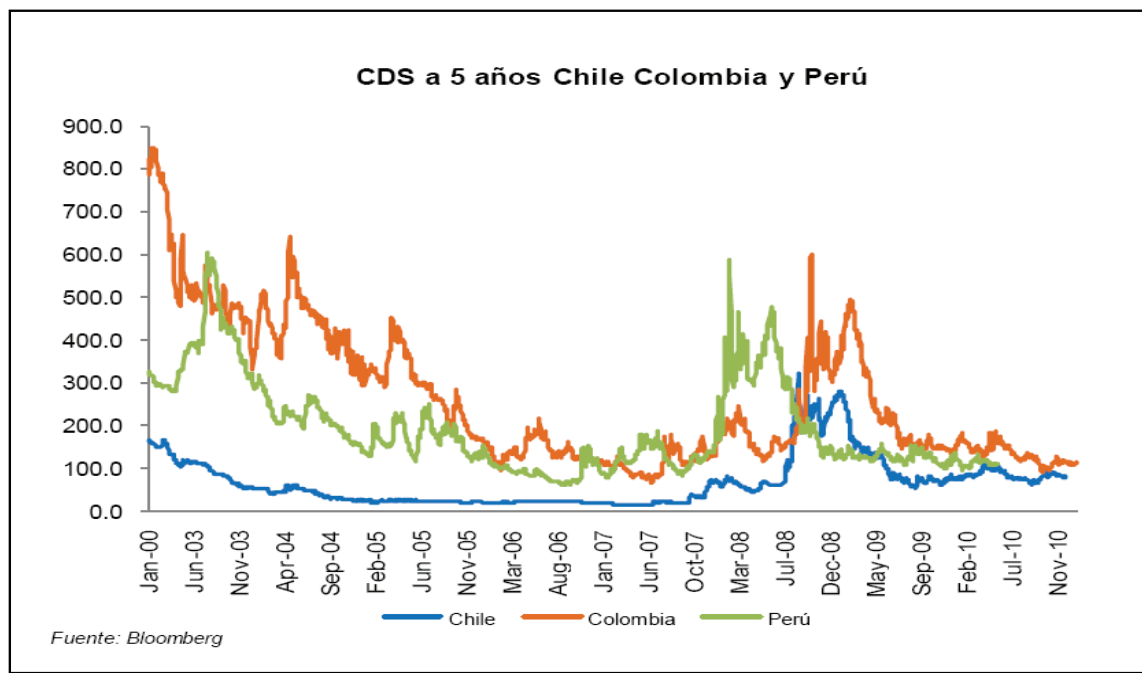

Gráfico 7

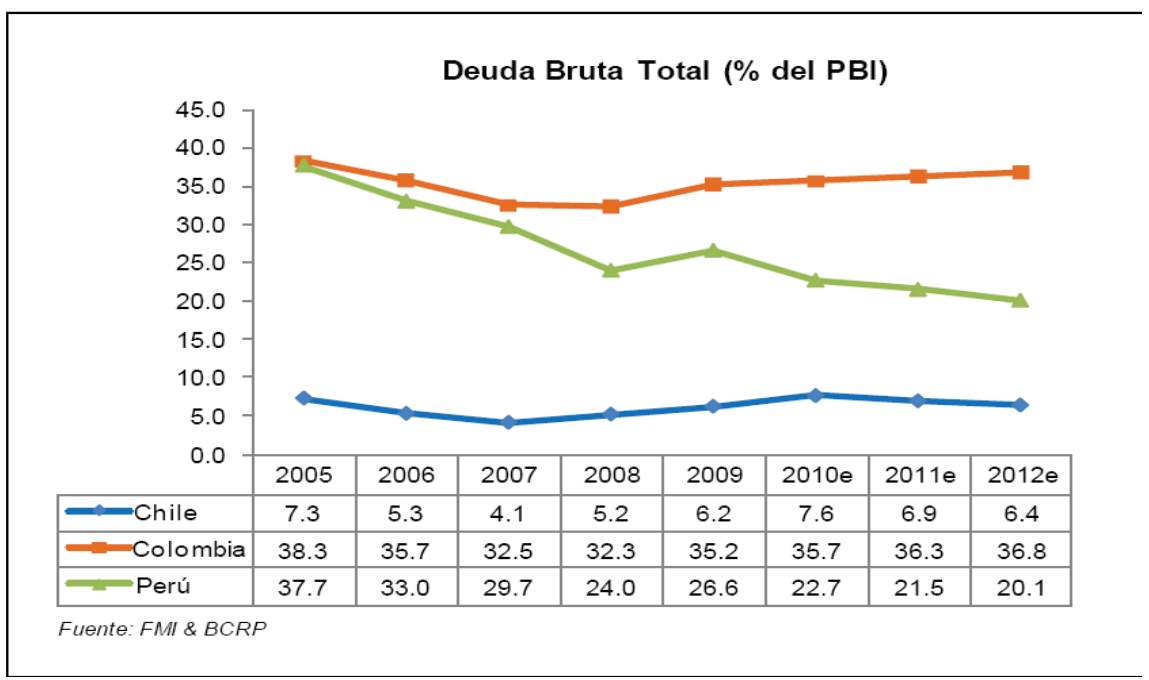

Gráfico 8 
EI Mercado Integrado Latino Americano (MILA). Contexto, Hipótesis y Reflexiones

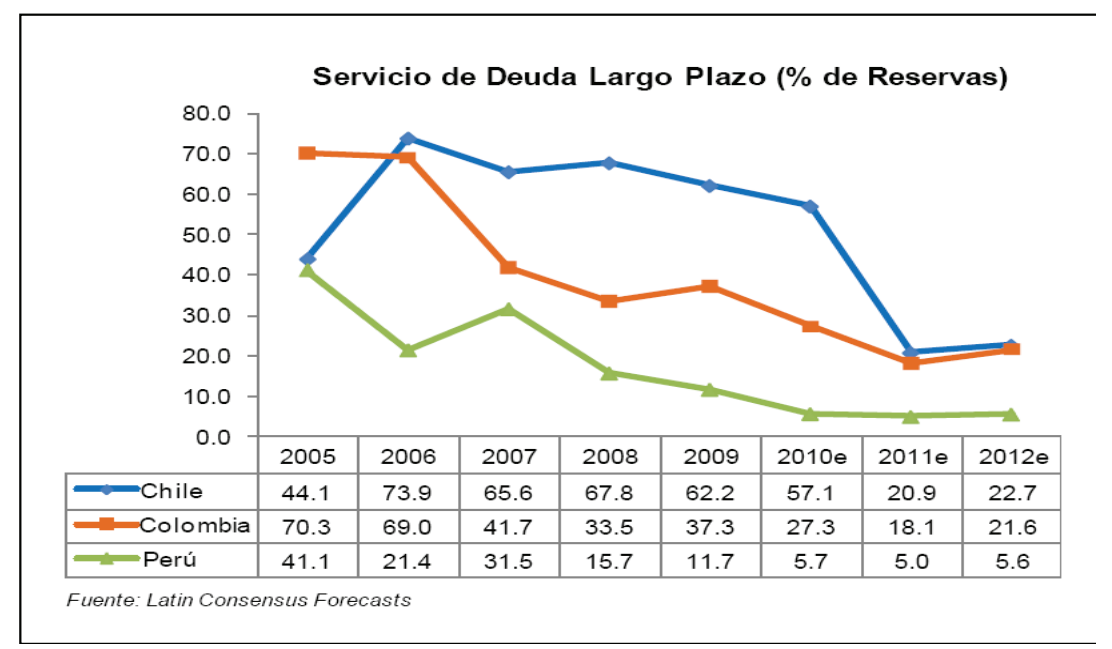

Gráfico 9

Cuadro 1

\begin{tabular}{|l|c|c|}
\hline \multicolumn{3}{|c|}{ Metas Explícitas de Inflación } \\
\hline & Meta de Inflación & Margen de Tolerancia \\
\hline Chile & $3.00 \%$ & $+/-1.00 \%$ \\
\hline Colombia & $3.00 \%$ & $+/-1.00 \%$ \\
\hline Perú & $2.00 \%$ & $+/-1.00 \%$ \\
\hline
\end{tabular}

Fuente: Inteligo $\mathrm{SAB}$

\section{Cuadro 2}

\begin{tabular}{|l|c|c|c|c|c|c|c|c|c|}
\hline \multicolumn{8}{|c|}{ Calificacions de Riesgo } \\
\hline & \multicolumn{3}{|c|}{2000} & \multicolumn{3}{c|}{2005} & \multicolumn{3}{c|}{$\mathbf{2 0 1 0}$} \\
\hline & Moody's & S\&P & Fitch & Moody's & S\&P & Fitch & Moody's & S\&P & Fitch \\
\hline Chile & Baa1 & A- & A- & Baa1 & A & A & Aa3 & A + & A \\
\hline Colombia & Ba2 & BB & BB + & Ba2 & BB & BB & Ba1 & BBB- & BB + \\
\hline Perú & Ba3 & BB- & BB & Ba3 & BB & BB & Baa3 & BBB- & BBB- \\
\hline
\end{tabular}

Fuente: Bloomberg 